\title{
Review on Antibiotic Resistance: Resistance Mechanisms, Methods of Detection and Its Controlling Strategies
}

\author{
Shimels Tikuye Yalew* \\ Animal Biotechnology Research Program, National Agricultural Biotechnology Research Centre, Ethiopian Institute of Agricultural \\ Research, Ethiopia \\ *Corresponding author: Shimels Tikuye Yalew, Animal Biotechnology Research Program, National Agricultural Biotechnology \\ Research Centre, Ethiopian Institute of Agricultural Research, Ethiopia
}

\begin{abstract}
ARTICLE INFO
Received: 慧 January 16, 2020

Published: 幽 January 24, 2020

Citation: Shimels Tikuye Yalew. Review on Antibiotic Resistance: Resistance Mechanisms, Methods of Detection and Its Controlling Strategies. Biomed J Sci \& Tech Res 24(5)-2020. BJSTR. MS.ID.004121.
\end{abstract}

Keywords: Antibiotic Resistance; Controlling Methods; Resistance mechanisms; Microorganisms; Oxazolidinones

\begin{abstract}
Antibiotic resistance (AR) is among the leading global health concerns to date. The misuse of antibiotics in the medical, veterinary and agricultural sectors, which include the inappropriate prescribing of antibiotics, their overuse in the livestock sector, and insufficient hygiene practices in hospital, all contribute to the rise of AMR. There are many mechanisms that bacteria exhibit to protect themselves from antibiotics and understanding the mechanisms by which bacteria resist antibiotics will become critical to solving the crisis. Laboratory based antibiotic resistance detection methods can determine resistance or susceptibility of an isolate against any therapeutic candidates. Appropriate use of antibiotics, vaccination, education, research, development of novel antibiotics, policy, regulations, surveillance of antimicrobial resistance and antibiotic use have a great role in minimization of antibiotic resistance.
\end{abstract}

Abbreviations: DNA: Deoxyribonucleic Acid; RNA: Ribonucleic Acid; AMR: Antimicrobial Agents; MRSA: Methicillin Resistant Staphylococcus Aurous; ABC: ATP Binding Cassette; MFS: Major Facilitator Superfamily; MATE: Multidrug and Toxic Effects; PBP: Penicillin Binding Protein; MIC: Minimum Inhibitory Concentration; MALDITOF MS: MatrixAssisted Laser Desorption Ionization-Time Of Flight Mass Spectrometry

\section{Introduction}

Antibiotics are used for treatment and prevention of bacterial infection. Antibiotics played a significant role in increasing life expectancy witnessed in the second half of the 20th century. Antibiotics transformed modern agriculture and livestock industries, the latter of which used antibiotics for prophylaxis, metaprophylaxis, treatment for infection, and as a growth promoter to enhance feed efficiency in healthy livestock [1]. Antibiotics are either cytotoxic or cytostatic to the micro-organisms, allowing the body's natural defenses, such as the immune system, to eliminate them. They are low molecular weight compounds and most of our natural products made by microorganisms or derived from natural products, which are active at low concentrations against other microorganisms but some antibiotics such as sulfa drugs and oxazolidinones do not originate from natural products [2]. Often, they act by inhibiting the synthesis of a bacterial cell, synthesis of proteins, deoxyribonucleic acid (DNA), ribonucleic acid (RNA), by a membrane disorganizing agent, or other specific actions [3]. Antibiotic resistance is among the leading global health concerns to date. Due to an overuse of antibiotics in medicine and agriculture, antibiotic resistance mechanisms commonly emerge and threaten modern medicine by diminishing the utility of clinically relevant antibiotics. Resistance to antimicrobial agents (AMR) has resulted in morbidity and mortality from treatment failures and increased health care costs [4].

Historically, veterinarians selected antimicrobials to treat bacterial infectious diseases based primarily on past clinical experiences. However, with the increase in bacterial resistance to traditionally used antimicrobials, it has become more difficult for 
clinicians to empirically select an appropriate antimicrobial agent [5]. Increasing global antimicrobial resistance (AMR) is a major threat to human and animal endangering decades of improvements in health-care outcomes. It endangers modern human and veterinary medicine and undermines food safety [6]. Conventional methods for phenotypic antibiotic susceptibility tests remain essential in routine diagnostics as they directly test for bacterial growth on solid or liquid media in the presence of antibiotics. Solid media based antibiotic susceptibility tests, such as disk diffusion assay or E-test, require at least 18-22 h for bacteria to grow visibly on agar plates to be able to evaluate growth inhibition with naked eye [7]. Molecular analysis can used to investigate the presence of an antimicrobial resistance gene [8]. Appropriate use of antibiotics, vaccination, education, research, development of novel antibiotics, policy, regulations, surveillance of antimicrobial resistance and antibiotic use have a great role in minimization of antibiotic resistance [9]. Therefore, the objective of the review is to review resistance mechanisms, methods of detection and its controlling strategies on antibiotic resistance.

\section{Antimicrobial Resistance}

Antimicrobial resistance (AMR) is a complex issue of global concern. The misuse of antibiotics in the medical, veterinary and agricultural sectors, which include the inappropriate prescribing of antibiotics, their overuse in the livestock sector, and insufficient hygiene practices in hospital, all contribute to the rise of AMR. Global trade and travel are also accelerating the spread. At the same time, the development pipeline of new antibiotics has slowed, mainly due to insufficient incentives, allowing microorganisms to outpace the development of new drugs. The discovery of antibiotics meant that previously lethal infections could be cured, and surgical procedures made safer, allowing for the development of modern medicine [10]. Micro-organisms are termed 'antimicrobial-resistant' or 'drugresistant' when they are no longer inhibited by an antimicrobial to which they were previously sensitive. Such resistance is called 'acquired resistance' and is encoded by resistance genes in the DNA of the microbe. Resistance genes can arise through spontaneous mutations in the microbial DNA, but some have evolved over many years due to natural selection by natural antimicrobials in the environment. These genes can antimicrobial resistance also transfer from drug-resistant microbes to drug sensitive ones. The first drug-resistant bacterium in a clinical setting was identified in the late 1940s; only 4 years after mass treatment with penicillin had been introduced. Since then the emergence and spread of drugresistant microbes has continued to grow. Highly resistant bacteria, such as methicillin resistant Staphylococcus aurous (MRSA) and vancomycin-resistant enterococci, account for a high percentage of hospital-acquired infections [11].

\section{Bacterial Resistance Mechanisms}

Bacteria have a remarkable genetic plasticity that allows them to respond to a wide array of environmental threats, including the presence of antibiotic molecules that may jeopardize their existence. Bacteria's are sharing the same ecological niche with antimicrobial-producing organisms have evolved ancient mechanisms to withstand the effect of the harmful antibiotic molecule and, consequently, their intrinsic resistance permits them to thrive in its presence [12]. There are many mechanisms that bacteria exhibit to protect themselves from antibiotics and understanding the mechanisms by which bacteria resist antibiotics will become critical to solving the crisis. Misuse of antibiotics may contribute to the development of resistant bacteria; an incomplete course of antibiotics risks not entirely eradicating the colony thus allowing the development of resistant bacteria. Mechanisms of drug resistance fall into several broad categories, including active efflux pumps, drug inactivation/alteration, modification of drug binding sites/targets, changes in cell permeability resulting in reduced intracellular drug accumulation, biofilm formation and others $[13,14]$.

\section{Efflux Pumps}

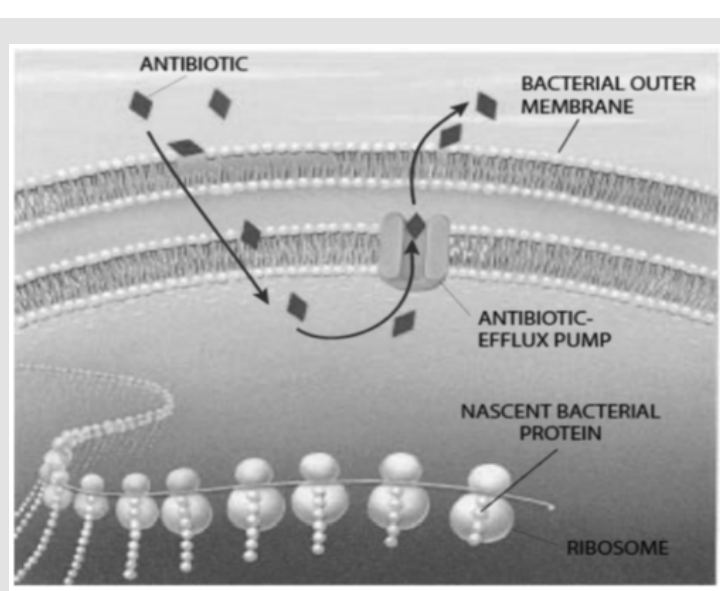

$\mathbf{A}$

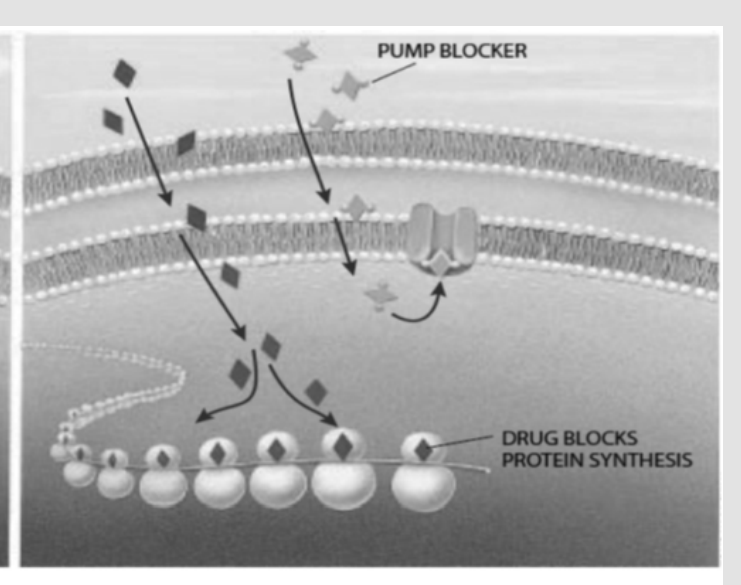

B

Figure 1: Bacterial efflux system A, system for antibiotic pumping out of the cell; B, antibiotic interfering with ribosomes in protein biosynthesis [17]. 
Efflux pumps are transporter proteins involved in the removal of toxic substances from the interior of the cell to the external environment. Efflux pumps in bacteria are major contributors to drug resistance; they extrude a broad spectrum of antibiotics to the exterior of the organism (Figure 1) [17]. Hence, infections caused by these pathogens can be difficult to treat [15]. Some efflux pumps are specific for a single drug while others are capable of transporting multiple substrates. There are five major efflux pump families: the small multidrug regulator subfamily (SMR), the Major Facilitator Superfamily (MFS), the ATP binding cassette (ABC) family, Resistance Nodulation Cell Division Subfamily (RND), and the Multidrug and Toxic Effects (MATE) family. A proton motive force mediated by the counter flow of protons drives the MFS, RND, MATE and SMR families. The genes of efflux pumps can be intrinsic or acquired. The intrinsic efflux mechanism of resistance is chromosomally encoded and is activated by environmental signals or by mutation in regulatory genes [16].

\section{Antibiotic Inactivation}

Bacteria uses several mechanisms of rendering antimicrobials inactive such as the enzymatic hydrolysis of antibiotics, group transfer and redox process. The production of $\beta$ - lactamases that hydrolyze the $\beta$-lactam ring of penicillins is the classical example of antibiotic inactivation. The enzymes can often be excreted by the bacteria, inactivating antibiotics before they reach their target within the bacteria. The second mechanism of antibiotic inactivation involves enzyme mediated structural alteration of the drug via transfer of a functional group such as an acyl, ribosyl, phosphoryl or thiol group. The modified antibiotic is unable to bind to the target due to the resultant change in the structure and the reaction is irreversible [18]. The third mechanism of antibiotic inactivation is by redox reaction [19].

\section{Target Modification}

Modification of the antibiotic target site makes the antibiotic unable to bind properly. Microorganisms cannot evade antimicrobial action by dispensing with them entirely because of the vital cellular functions of the target sites. In this mechanism, bacteria found ways to alter the targets of antimicrobial agents. The classical example of drug target modification is the staphylococcal mechanism of variously altering the Penicillin Binding Protein (PBP) which is the target of $\beta$-lactam antibiotics [20].

\section{Reducing Entry of Antimicrobial Agents}

Mostly antimicrobial compounds always require access into the bacterial cell to reach their target site. Porin channels are the passageways by which antibiotics normally cross the bacterial outer membrane. Some bacteria protect themselves by prohibiting these antimicrobial compounds from entering to their cell walls [21].

\section{Mutation}

Mutation is a spontaneous change in the DNA sequence within the gene that may lead to a change in the trait which it codes for (Figure 2) [14]. A single base pair change may lead to a corresponding change in one or more of the amino acids for which its codes, which can then change the enzyme or cell structure that consequently changes the affinity or effective activity of the targeted antimicrobials. In prokaryotic genomes, mutations frequently occur due to base changes caused by exogenous agents, DNA polymerase errors, deletions, insertions and duplications [22].

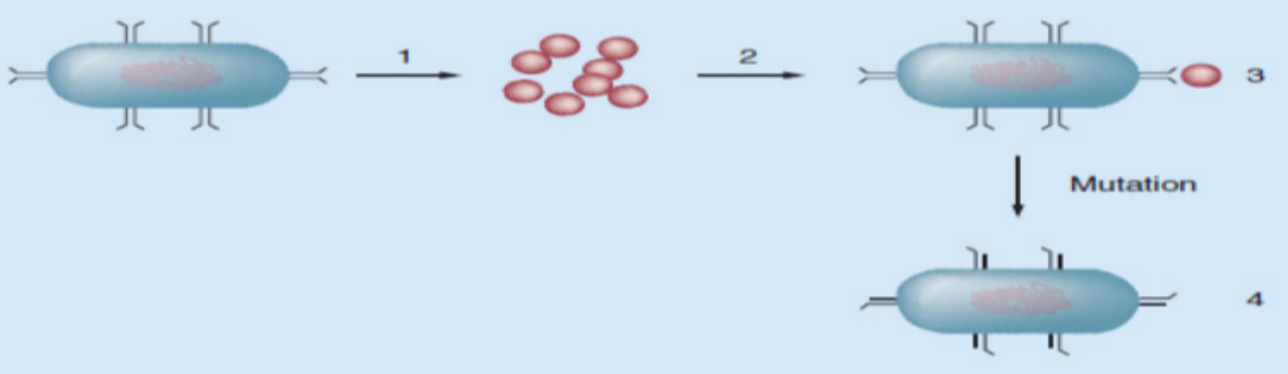

Figure 2: A diagram to show how mutations lead to antibiotics being unable to bind to their target.

a) A wild-type bacteria.

b) Antibiotics that would bind to and destroy the target bacteria.

c) The antibiotic is able to bind to and destroy the target wild-type bacteria.

d) After mutation occurs, the binding site is altered, and the antibiotic is unable to bind to the mutant bacteria and is unable to kill it. These bacteria will proliferate creating a new resistant colony [14].

\section{Biofilm Formation}

Complex microbial communities containing bacteria and fungi are called biofilms. Microorganisms synthesize and secrete a protective matrix that attaches the biofilm firmly to a living or non- living surface. A biofilm can be described as bacteria embedded in a thick, slimy barrier of sugars and proteins. The biofilm barrier protects the microorganisms from external threats. The high cell density in biofilms increases the absolute numbers of resistant mutants that can be selectable under antimicrobial pressure [23]. 


\section{Detecting Methods of Antimicrobial Resistance}

Antimicrobial susceptibility testing methods are in vitro procedures used to detect antimicrobial resistance in individual bacterial isolates. Those laboratory-based detection methods can determine resistance or susceptibility of an isolate against any therapeutic candidates. Those methods can also be used for monitoring the emergence and spread of resistant microorganisms in the population [24]. Guidelines and recommendations for these are continuously updated by certain organizations worldwide, those which specify antimicrobial testing methods and interpretative criteria for veterinary pathogens are: the CLSI in the USA, OIE in EU and CDS-AST in Australia [25]. Among the available tests, agar disk-diffusion and the broth micro dilution methods are the two most commonly used methods in veterinary laboratories. Some examples of antibiotic sensitivity testing methods are dilution method (broth and agar dilution method), disk-diffusion method, E-test, automated methods and genotypic methods [26].

\section{Disk-diffusion method}

The application of commercially available drug-impregnated filter paper disks to the surface of an agar plate that has been inoculated to confluence with the organism of interest is called disk diffusion [27]. Disk diffusion is also known as Kirby-Bauer antibiotic testing. The drug diffuses radially through the agar, the concentration of the drug decreasing logarithmically as the distance from the disk increases and results in a circular zone of growth inhibition around the disk, the diameter of which is inversely proportional to the MIC. The zone diameters are interpreted on the basis of guidelines published by CLSI, and the organisms are reported as susceptible, intermediate, or resistant. Disk diffusion can only be used to test rapidly growing organisms, for which criteria for interpretation of zone sizes are available [28]. The diameter of zone of inhibition around the antimicrobial disk is related to minimum inhibitory concentration (MIC) for that particular bacterial isolate; the zone of inhibition correlates inversely with the MIC of the test bacterium. Generally, having larger the zone of inhibition, the lower the concentration of antimicrobial required to inhibit the growth of the organisms [5].

Disk diffusion test is performed by applying a bacterial inoculum of approximately $1-2 \times 108 \mathrm{CFU} / \mathrm{ml}$ to the surface of a large $(150 \mathrm{~mm}$ diameter) Mueller-Hinton agar plate. Zones of growth inhibition around each of the antibiotic disks are measured to the nearest millimeter. The diameter of the zone shows the susceptibility of the isolate and the diffusion rate of the drug through the agar medium. The zone diameters of each drug are interpreted using the criteria published by the Clinical and Laboratory Standards Institute (CLSI0. The results of disk diffusion test are "qualitative," in that a category of susceptibility (i.e., susceptible, intermediate, or resistant) is derived from the test rather than an MIC [24]. The test is straightforward to perform, reproducible, and does not require expensive equipment. The diameter of the zone of growth inhibition is measured with calipers or a ruler and recorded in millimeters [29].

\section{Dilution Method}

Agar dilution and broth dilution are the most commonly used methods to determine the minimal concentration of antimicrobial agents that kill (bactericidal activity, MBC) or inhibit the growth (bacteriostatic activity, MIC) of microorganisms. Dilution methods are performed when quantitative methods are required for microorganisms with a variable growth rate [26].
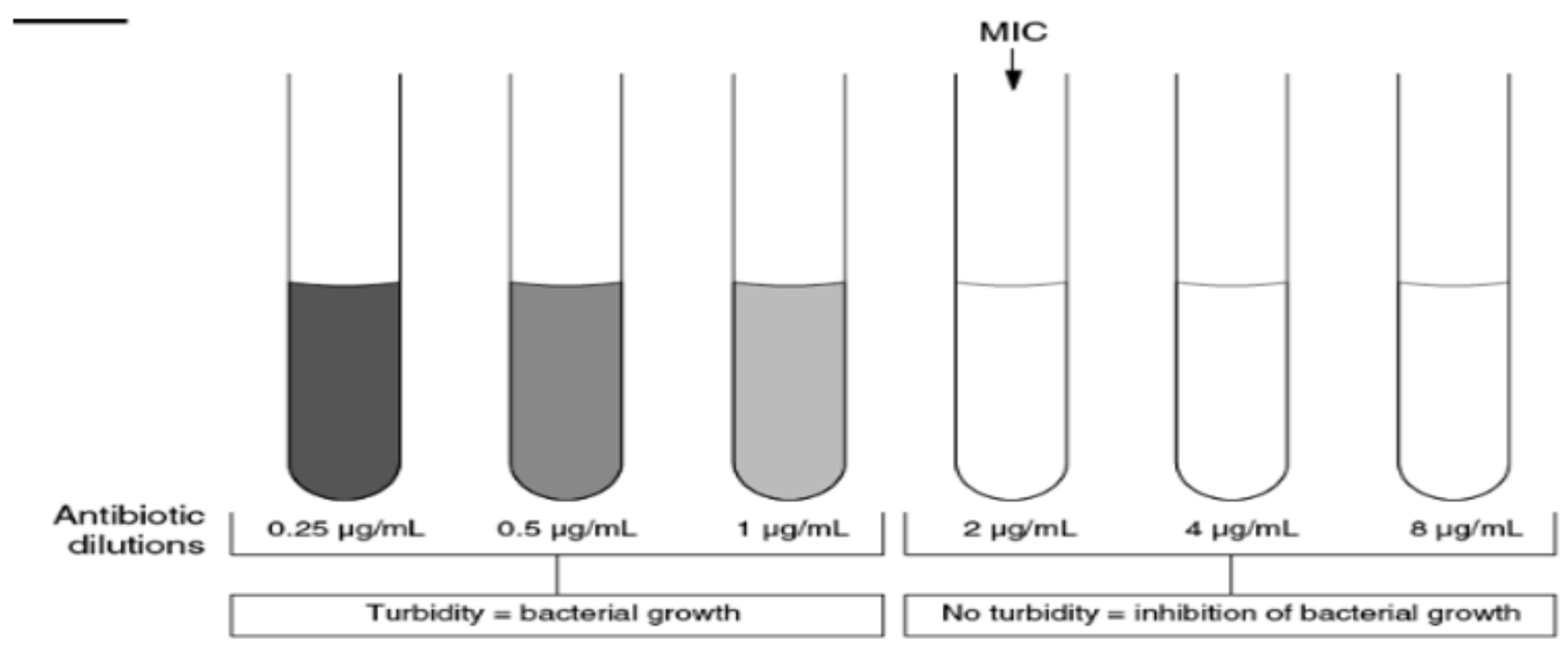

Figure 3: Diagram of broth dilution method [30]. 
Broth Dilution Technique: The Broth dilution method involves subjecting the isolate to a series of concentrations of antimicrobial agents in a broth environment. Micro dilution testing uses about 0.05 to $0.1 \mathrm{ml}$ total broth volume and can be conveniently performed in a micro titer format. Macro dilution testing uses broth volumes at about $1.0 \mathrm{ml}$ in standard test tubes. For both broth dilution methods, the lowest concentration at which the isolate is completely inhibited is recorded as the minimal inhibitory concentration or MIC (Figure 3) [30]. The MIC is thus the minimum concentration of the antibiotic that will inhibit this isolate. The broth dilution technique of antibiotic susceptibility testing is also known as the minimal inhibitory concentration (MIC) technique. Test tubes or wells containing increasing concentrations of each antibiotic to be tested, from 0.0312 to $512 \mu \mathrm{g} / \mathrm{ml}$, are inoculated with a fixed volume of nutrient broth containing a standard concentration of bacteria. The concentration of the antibiotic in each tube is double that in the previous tube [30]. In the broth dilution assay, an antimicrobial is added to a culture tube of nonselective broth medium at different concentrations [31]. Tubes are incubated under optimum conditions for the test microorganism from 16 to 24 hours. Antimicrobial effect could be determined by spectro-photometry or by plating counting [32].

The Agar Dilution Method: Agar dilutions are most often prepared in petri dishes and have advantage that it is possible to test several organisms on each plate [33]. In the agar dilution method, the antimicrobial agent is incorporated into the agar medium with each plate containing a different concentration of the agent. The inoculum can be applied rapidly and simultaneously to the agar surfaces using an inoculum replicating apparatus. Mueller-Hinton agar is prepared from a dehydrated base. The advantages of agar dilution testing include the reproducible results and satisfactory growth of most nonfastidious organisms. Agar dilution testing generally is not performed in routine clinical laboratories but can be ideal for regional reference laboratories or research laboratories that must test large numbers of isolates [34].

\section{Epsilometer Test (E-Test)}

Epsilometer test (E- test) is an 'exponential gradient' method of determination of antimicrobial resistance. The E-test is developed to provide a direct quantification of antimicrobial susceptibility of microorganisms. This is a quantitative method that applies both the dilution of antibiotic and diffusion of antibiotic into the medium. The device consists of a predefined, continuous, and exponential gradient of antibiotic concentrations immobilized along a rectangular plastic test strip [35]. The principle of E test method is based on antimicrobial concentration gradient in an agar plate (Figure 4) [27]. These strips are impregnated on the underside with a dried antibiotic concentration gradient and are labeled on upper surface with a concentration scale. When this E test strip was placed onto an inoculated agar plate, there was an immediate release of the drug. E test have been used to determine MIC for fastidious organisms like S. pneumoniae, $\beta$-hemolytic streptococci, N.gonorrhoeae, Haemophilus sppcies and anaerobes. The cost the E test is little more when compared to disk diffusion method. However, the E test is simple, accurate, and reliable and is also used to determine the Minimum Inhibitory Concentration (MIC) of antifungal agents and antimycobacterial agents [33].

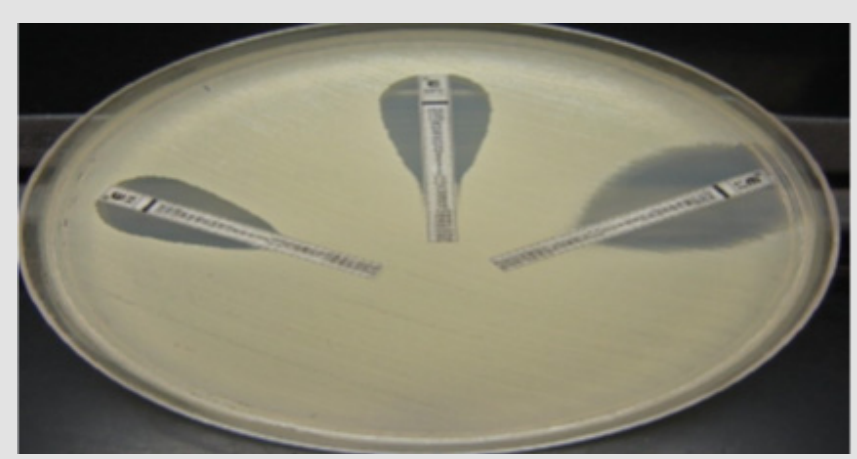

Figure 4: E Test gradient diffusion method [27].

\section{Automated Instrument Methods}

There are a variety of commercially available automated systems available to help reduce the technical time required to perform and record routine sensitivity tests. For example, the results of disk sensitivity tests and breakpoint sensitivity tests can be read using a camera interfaced to a computer system. Other Systems utilize liquid cultures and detect the effect of antibiotics on the rate of bacterial growth through measurement of turbidity (nephelometry) or the production of $\mathrm{CO}_{2}$. These automated systems can significantly shorten the necessary incubation time [36]. There are different types of automated instruments (Micro Scan Walk away, BD phoenix, Trek Sensititere and Vitek 1 and Vitek 2). The advantage of using this test is increased reproducibility, decreased labor costs and issued rapid results but the disadvantage is they are not available widely in developing country including Ethiopia [37].

\section{Molecular Methods for Detection of Antimicrobial Resistance}

Molecular characterization of the genetic mechanism(s) underlying a given phenotypic result, obtained by traditional antimicrobial sensitivity testing, is now an integral part of many clinical investigations in relation to bacterial infections, whether in humans or animals. In some cases, when phenotypic results are too time-consuming, non-conclusive, or unavailable, molecular analysis can be used to investigate the presence of a given gene. Molecular methods are being used extensively by both research and reference laboratories. Some of the methods employed, such as PCR and hybridization techniques, have been used for decades, while new methods such as Whole-Genome Sequencing (WGS) and Matrix-Assisted Laser Desorption Ionization-Time of Flight Mass Spectrometry (MALDITOF MS) are just emerging [8].

\section{Strategies to Minimize Antibiotic Resistance}

Antibiotic-resistant bacteria are spread globally in the same way as other bacteria. This means they can be transferred between people, animals and foodstuffs, and they can spread in 
our environment. These links between the various sectors mean that efforts to combat antibiotic resistance must be made from a broad perspective. Prudent use of antibiotics in healthcare, animal health and agricultural settings is essential to slow the emergence of resistance and extend the useful lifetime of effective antibiotics. Treatment conditions of antibiotics for bacterial infections have gradually worsened, because of antibiotic resistance and the lack of new drugs. Apparently, it is now clear that antibiotic use can increase the emergence of antibiotic-resistant bacteria, and reducing prescribing is one of the effective ways to reduce selection pressure [38]. Resistant bacteria can spread quickly and represent a reservoir of bacteria that can spread further to both people and animals. Animals can get infections that are difficult to treat as a result of resistant bacteria and causes suffering for the animals and economic losses.

To prevent overuse and misuse of antibiotics, a formalized, practical guideline for appropriate antibiotic prescribing should be developed and followed by formulary implementation of the guidelines contained therein. The development of quick and effective molecular diagnostic techniques for identification and epidemiological surveillance of resistance genes of antibioticresistant pathogens can improve current control strategies. Reducing use of antibiotic in agriculture, especially in food animals, is also important. Changing policies to use of antibiotics in livestock can include ban or restriction on using veterinary important antibiotics, promising financial incentives for developing livestockspecific antibiotics, making drug licensing rules more stringent and imposing penalties. Effective strategies are required to address the problem of antibiotic resistance. Therefore, appropriate use of antibiotics, vaccination, education, research, development of novel antibiotics, policy, regulations, surveillance of antimicrobial resistance and antibiotic use have a great role in minimization of antibiotic resistance (Figure 5)[9,39].

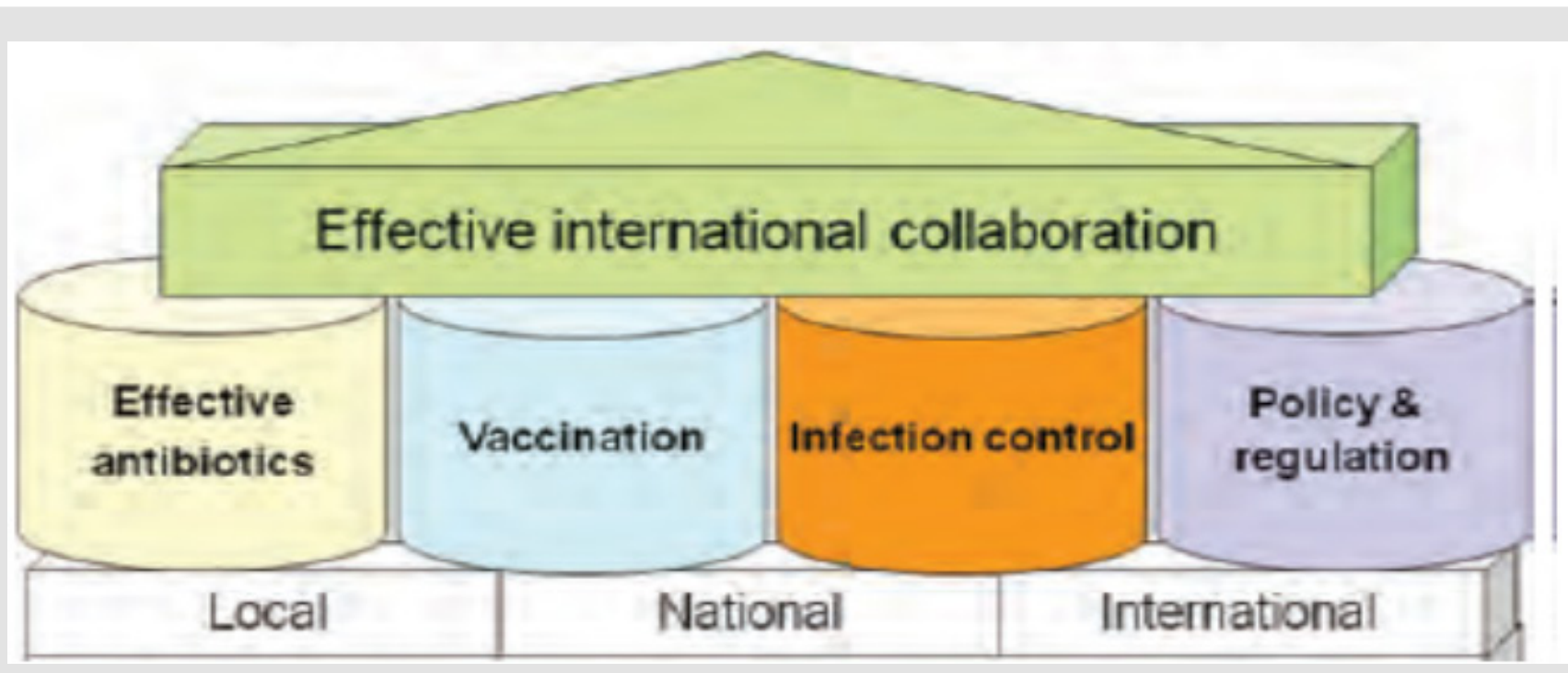

Figure 5: Major components of strategies to minimize antibiotic resistance [39].

\section{Conclusion}

Antibiotic resistance is rising to dangerously high levels in all parts of the world. New resistance mechanisms are emerging and spreading globally, threatening our ability to treat common infectious diseases. Infections caused by antibiotic-resistant germs are difficult, and sometimes impossible, to treat. Therefore, prudent use of antibiotics in healthcare, animal health and agricultural settings is essential to slow the emergence of resistance and extend the useful lifetime of effective antibiotics.

\section{Acknowledgements}

I would like to express my heartily thanks to my families especially my lovely wife and my baby son Yeab Sira Shimels for giving me a time to prepare the manuscript.

\section{References}

1. Qiao M, Ying GG, Singer AC, Zhu YG (2018) Review of antibiotic resistance in China and its environment. Environ Int 110: 160-172.

2. Martens E, Demain AL (2017) The antibiotic resistance crisis, with a focus on the United States. J Antibiot 70(5): 520-526.

3. Zaman S Bin, Hussain MA, Nye R, Mehta V, Mamun KT, et al. (2017) A review on antibiotic resistance: alarm bells are ringing. Cureus 9(6): e1403.

4. Avesar J, Rosenfeld D, Truman Rosentsvit M, Ben Arye T, Geffen Y, et al. (2017) Rapid phenotypic antimicrobial susceptibility testing using nanoliter arrays. Proc Natl Acad Sci 114(29): E5787-E5795.

5. White DG, Acar J, Anthony F, Franklin A, Gupta R, et al. (2001) Antimicrobial resistance: standardisation and harmonisation of laboratory methodologies for the detection and quantification of antimicrobial resistance. Rev Sci Tech Int des Epizoot 20(3): 849-855. 
6. Gay N, Belmonte O, Collard JM, Halifa M, Issack MI, et al. (2017) Review of antibiotic resistance in the Indian Ocean Commission: A human and animal health issue. Front public Heal 5: 162.

7. Veses Garcia M, Antypas H, Löffler S, Brauner A, Andersson Svahn H, et al. (2018) Rapid Phenotypic Antibiotic Susceptibility Testing of Uropathogens Using Optical Signal Analysis on the Nanowell Slide. Front Microbiol 9.

8. Anjum MF, Zankari E, Hasman H (2017) Molecular Methods for Detection of Antimicrobial Resistance. Microbiology 5(6).

9. Laxminarayan R, Duse A, Wattal C, Zaidi AKM, Wertheim HFL, et al. (2013) Antibiotic resistance-the need for global solutions. Lancet Infect Dis 13(12): 1057-1098.

10. Merrett GLB (2013) Tackling antibiotic resistance for greater global health security. Chatham House.

11. Holmes AH, Moore LSP, Sundsfjord A, Steinbakk M, Regmi S, et al. (2016) Understanding the mechanisms and drivers of antimicrobial resistance. Lancet 387(10014): 176-187.

12. Munita JM, Arias CA (2016) Mechanisms of antibiotic resistance. Microbiol Spectr 4(2).

13. Wilson DN (2014) Ribosome-targeting antibiotics and mechanisms of bacterial resistance. Nat Rev Microbiol 12(1): 35-48.

14. Ali J, Rafiq QA, Ratcliffe E (2018) Antimicrobial resistance mechanisms and potential synthetic treatments. Futur Sci OA 4(4).

15. McKeegan KS, Borges Walmsley MI, Walmsley AR, (2003) The structure and function of drug pumps: an update. Trends Microbiol 11(1): 21-29.

16. Lomovskaya O, Watkins $\mathrm{W}$ (2001) Inhibition of efflux pumps as a novel approach to combat drug resistance in bacteria. J. Mol. Microbiol Biotechnol 3(2): 225-236.

17. Giedraitienè A, Vitkauskienè A, Naginienè R, Pavilonis A (2011) Antibiotic resistance mechanisms of clinically important bacteria Medicina (B. Aires) 47(3): 137-146.

18. Kumar S, Varela MF (2013) Molecular mechanisms of bacterial resistance to antimicrobial agents. Chemotherapy 522-534.

19. Džidić S, Šušković J, Kos B (2008) Antibiotic resistance mechanisms in bacteria: biochemical and genetic aspects. Food Technol Biotechnol 46(1): 11-21

20. Davies J, Davies D (2010) Origins and evolution of antibiotic resistance. Microbiol. Mol Biol Rev 74(3): 417-433.

21. Poole K (2002) Mechanisms of bacterial biocide and antibiotic resistance. J Appl Microbiol 92: 55S-64S

22. Martinez JL, Baquero F (2000) Mutation frequencies and antibiotic resistance. Antimicrob. Agents Chemother 44(7): 1771-1777.

23. Soto SM (2013) Role of efflux pumps in the antibiotic resistance of bacteria embedded in a biofilm. Virulence 4(3): 223-229.

\section{ISSN: 2574-1241}

DOI: $10.26717 /$ BJSTR.2020.24.004121

Shimels Tikuye Yalew. Biomed J Sci \& Tech Res

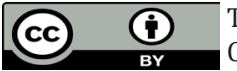

This work is licensed under Creative

Commons Attribution 4.0 License

Submission Link: https://biomedres.us/submit-manuscript.php
24. Jorgensen JH, Turnidge JD (2015) Susceptibility test methods: dilution and disk diffusion methods. Eleventh Edition American Society of Microbiology 1253-1273.

25. Guetaba MY (2015) Prevalence and Antibiotic Resistance of Salmonella Sp., Shigella Sp. and Escherichia Coli in Fresh Retail Chicken in the Accra Metropolis. University of Ghana Digital collections.

26. Balouiri M, Sadiki M, Ibnsouda SK (2016) Methods for in vitro evaluating antimicrobial activity: A review. J Pharm Anal 6(2): 71-79.

27. Patel RM (2012) The guiding principles on antimicrobial susceptibility testing. Bull Pharm Res 2(3): 146-153.

28. Jones RN, Ballow CH, Biedenbach DJ, Pettis Memorial VA (2001) Multilaboratory assessment of the linezolid spectrum of activity using the Kirby-Bauer disk diffusion method: Report of the Zyvox® Antimicrobial Potency Study (ZAPS) in the United States. Diagn Microbiol Infect Dis 40(1-2): 59-66

29. Tendencia EA, Lila Ruangpan (2004) Laboratory Manual of Standardized Methods for Antimicrobial Sensitivity Tests for Bacteria Isolated from Aquatic Animals and Environment. Aquaculture Department Southeast Asian Fisheries Development Center 13-29.

30. Pierce Hendry SA, Dennis J (2010) Bacterial culture and antibiotic susceptibility testing. Compend Contin Educ Vet 32(7): 1-5.

31. Chow VTK, Inglis TJJ, Song KP (2006) Diagnostic clinical microbiology. World Scientific 539-592.

32. Wiegand I, Hilpert K, Hancock REW (2008) Agar and broth dilution methods to determine the minimal inhibitory concentration (MIC) of antimicrobial substances. Nat Protoc 3(2): 163-175.

33. Lalitha MK (2004) Manual on antimicrobial susceptibility testing Perform. Stand. Antimicrob. Test Twelfth Informational Suppl 454-456.

34. Luber P, Bartelt E, Genschow E, Wagner J, Hahn H (2003) Comparison of broth microdilution, E Test, and agar dilution methods for antibiotic susceptibility testing of Campylobacter jejuni and Campylobacter coli. J Clin Microbiol 41(3): 1062-1068.

35. Ncube NS, Afolayan AJ, Okoh AI (2008) Assessment techniques of antimicrobial properties of natural compounds of plant origin: current methods and future trends. African J Biotechnol 7(12).

36. Jorgensen JH, Ferraro MJ (2009) Antimicrobial susceptibility testing: a review of general principles and contemporary practices. Clin Infect Dis 49(11): 1749-1755.

37. Clark AW, Gladnick P, Armstrong RE, Bachur N, Berndt KW, et al. (2000) Automated microbiological testing apparatus and methods therefor.

38. Lee CR, Cho IH, Jeong BC, Lee SH (2013) Strategies to minimize antibiotic resistance. Int J Environ Res Public Health 10(9): 4274-4305.

39. Tillotson G (2015) Antimicrobial resistance: what's needed. Lancet Infect Dis 15(7): 758-760.

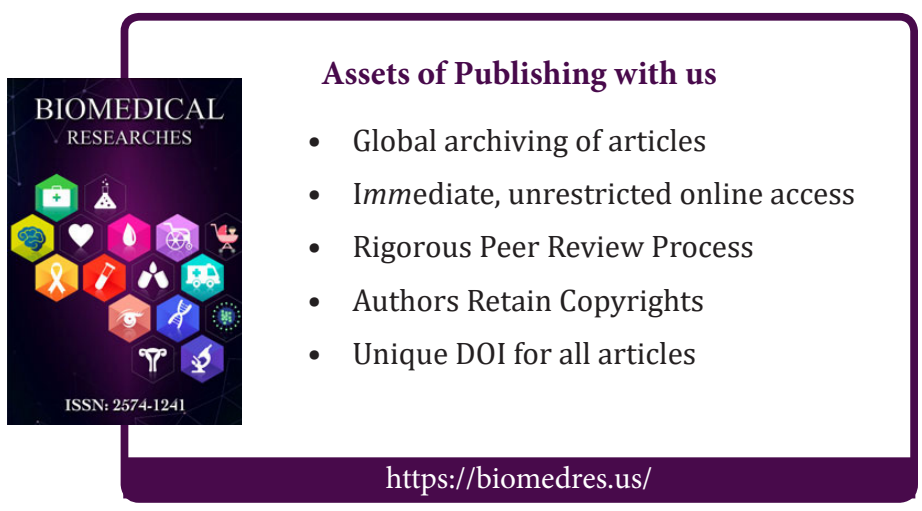

Copyright@ Shimels Tikuye Yalew | Biomed J Sci \& Tech Res | BJSTR. MS.ID.004121. 